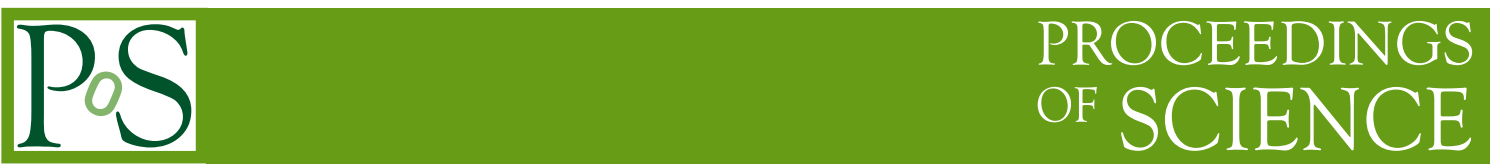

\title{
Minimum Bias Trigger in ATLAS
}

\section{Regina E. Kwee* on behalf of the ATLAS Collaboration}

Institut für Physik, Humboldt-Universität zu Berlin, Germany

CERN, 1211 Geneva 23, Switzerland

E-mail: Regina.Kweedcern.ch

Since the restart of the LHC in November 2009, ATLAS has collected inelastic $p p$-collisions to perform measurements on charged-particle densities. Experimental data is needed to constrain various phenomenological models of soft parton interactions. Understanding the trigger efficiencies for different event types is therefore crucial to minimize any possible bias in the event selection. ATLAS uses two main minimum bias triggers, featuring complementary detector components and trigger levels. These triggers are a hardware based first level trigger covering $2.09<$ $|\eta|<3.84$ which has proven to select $p p$-collisions very efficiently and the Inner Detector based minimum bias trigger using a random trigger on filled bunches and central tracking detectors for the event selection. Both triggers were essential for the analysis of general properties of charged particles at LHC energies. Their performance and trigger efficiency measurements as well as studies on possible bias sources are presented.

35th International Conference of High Energy Physics

July 22-28, 2010

Paris, France

* Speaker. 


\section{Introduction and Motivation}

During one year of LHC operation from November 2009, the ATLAS detector [1] at the LHC has routinely recorded data from proton-proton $(p p)$ collisions at the centre-of-mass energy of $\sqrt{s}=900 \mathrm{GeV}, 2.36$ and $7 \mathrm{TeV}$. The rapidly increasing luminosity has allowed for fast progress in early physics analyses towards the re-discovery of Standard Model processes and enabled commissioning of crucial tools for physics searches beyond the Standard Model. The hadronic environment at the LHC, in particular at design energy of $\sqrt{s}=14 \mathrm{TeV}$ and luminosity of $10^{34} \mathrm{~cm}^{-2} \mathrm{~s}^{-1}$, make searches for rare new physics events especially challenging. In the high luminosity regime several soft inelastic interactions are expected to overlap with hard scattering processes forming socalled pile-up within one bunch-crossing (BC). It is vital to first understand such an environment with highest possible precision. The measurements of charged-particle multiplicities from $p p$ interactions are an important step towards this understanding, eventually improving the modeling of these processes as theoretical predictions at LHC energies are highly uncertain. Such measurements were performed at all three centre-of-mass energies $[2,4]$ on data that were collected by a dedicated setup of minimum bias triggers. The deployment of such triggers at initial luminosities of $\mathscr{L}=10^{27}-10^{30} \mathrm{~cm}^{-2} \mathrm{~s}^{-1}$ was crucial as the interaction probability per BC was significantly smaller than $50 \%$. For characterisation of inelastic processes it is vital that these triggers introduce indeed only minimal bias in the event selection.

\section{Minimum Bias Triggers in ATLAS and Performance Results}

ATLAS has several complementary minimum bias (MB) triggers making use of the three levels of the trigger system [1]: Level-1 (L1), running custom-made hardware triggers but housing also internal triggers like random or clock triggers, and the high-level trigger (HLT) featuring two more levels, Level-2 (L2) and Event Filter (EF), both selecting on software derived decisions.

During initial operation ATLAS selected inelastic $p p$ interactions using the Minimum Bias Trigger Scintillators (MBTS), which provide a hardware L1 trigger. The MBTS cover a pseudorapidity range of $2.09<|\eta|<3.84$ and provide full coverage of the azimuthal angle $\phi$. These plastic scintillator counters are segmented into two units in $\eta$ and eight units in $\phi$ and positioned $3.56 \mathrm{~m}$ away from the interaction point on each side. Trigger signals from the MBTS are discriminated and used to form multiplicity constraints. With the total of 32 read-out channels, the MBTS represents a relative simple system which played also a keyrole in the ATLAS detector commissioning thanks to its high sensitivity to proton interactions. Using the lowest trigger configuration, data for several analyses $[4,5]$ were selected by requiring at least one counter to be above threshold (L1_MBTS_1). Other configurations, which served for efficiency determination or as fall-back triggers in case of higher beam background, were also enabled, e.g. requiring at least two hits in total (L1_MBTS_2) or a coincidence of hits on each side, e.g. one per side (L1_MBTS_1_1).

A complementary approach is realised by an Inner Detector Minimum Bias trigger (ID Mb) with selection at HLT. A random trigger on colliding bunches is employed at L1 while the selection at L2 is based on hit cluster multiplicities in the silicon tracking detectors, i.e. in the Pixel and Semiconductor tracker (SCT), both covering $|\eta|<2.5$. To efficiently reject emtpy BC events, pixel clusters have to contain sufficient energy deposition while the micro-strips in the SCT are 
required to overlap in $\eta$ and $\phi$. At the EF a functionality of rough silicon track reconstruction can be enabled in case beam induced background needs to be suppressed online. During the 2010 data-taking, this additional selection step was not necessary due to very good vacuum conditions.

Performance Results For the charged-particle multiplicity measurements the trigger performance of MBTS and ID Mb were studied on data recorded at $\sqrt{s}=900 \mathrm{GeV}$ and $7 \mathrm{TeV}$ [3]. Offline selection criteria were applied that comprised of track quality criteria e.g. on the track length or on impact parameters to exclude tracks from possible beam-gas interactions. The trigger efficiency of ID Mb showed no dependency as a function of track multiplicity, see Fig. 1(a), which was determined in data using L1_MBTS_2 as a control trigger. Consequently, ID Mb introduces no bias in $p_{\mathrm{T}}$ or $\eta$ either. This made ID Mb an excellent control trigger for the measurement of the trigger efficiency of L1_MBTS_1. Possible correlation of ID Mb and L1_MBTS_1 was investigated in $\mathrm{MC}$ and was found to be negligible within statistical uncertainties. Also, no bias in $\eta$ and $p_{\mathrm{T}}$ was observed within uncertainties [3], merely a small dependency of L1_MBTS_1 was found for low multiplicities as shown in Fig. 1(b). The ATLAS minimum bias triggers have been successfully used for initial $p p$ physics studies at centre-of-mass energies of $900 \mathrm{GeV}, 2.36 \mathrm{TeV}$ and $7 \mathrm{TeV}$.
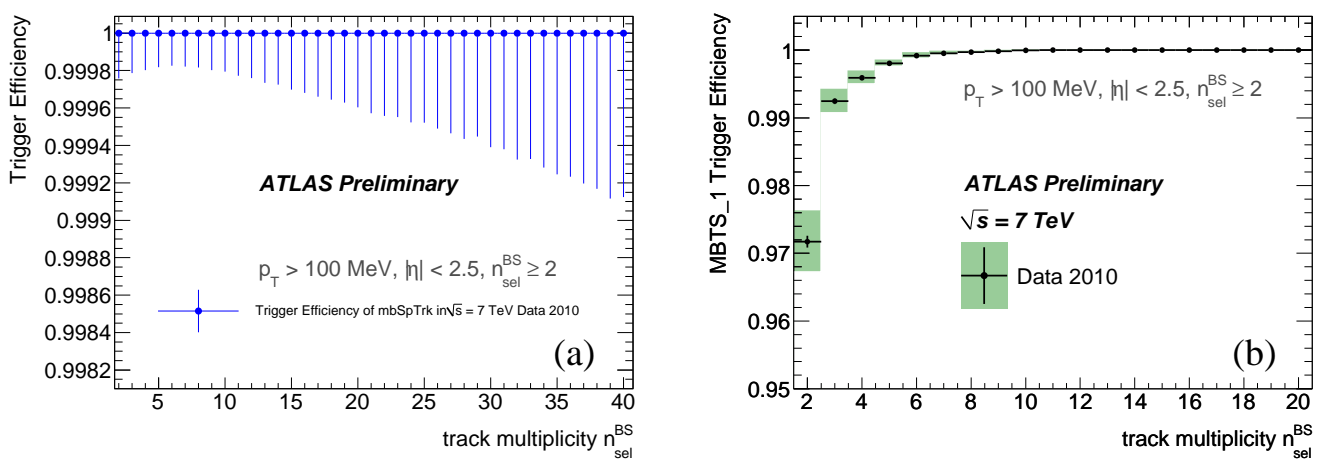

Figure 1: Trigger efficiency measured in data at $\sqrt{s}=7 \mathrm{TeV}$ of ID Mb (also named mbSpTrk) w.r.t. L1_MBTS_2 (a) and L1_MBTS_1 w.r.t. ID Mb (b). Both measurements were made in the indicated phasespace which was used for [4]. The error bars in (a,b) show statistical uncertainties only while the band in (b) contains systematic and statistical uncertainties.

\section{References}

[1] The ATLAS Collaboration, The ATLAS Experiment at the CERN Large Hadron Collider, 2008 JINST $3 \mathrm{~S} 08003$

[2] The ATLAS Collaboration, Charged-particle multiplicities in pp interactions at $\sqrt{s}=900 \mathrm{GeV}$ measured with the ATLAS detector at the LHC, Phys Lett B 688 (2010) Issue 1, 21-42

[3] The ATLAS Collaboration, Performance of the Minimum Bias Trigger in p-p Collisions at $\sqrt{s}=7 \mathrm{TeV}$, ATLAS-CONF-2010-068, July 2010

[4] The ATLAS Collaboration, Charged-particle multiplicities in pp interactions for track $p_{T}>100 \mathrm{MeV}$ at $\sqrt{s}=0.9$ and $7 \mathrm{TeV}$ measured with the ATLAS detector at the LHC, ATL-CONF-2010-046, Jul. 2010

[5] The ATLAS Collaboration Track-based underlying event measurements in pp collisions at $\sqrt{s}=900 \mathrm{GeV}$ and $7 \mathrm{TeV}$ with the ATLAS Detector at the LHC, ATLAS-CONF-2010-081, Aug. 2010 